\title{
LINEARIZATION OF CERTAIN UNIFORM HOMEOMORPHISMS
}

\author{
ANTHONY WESTON \\ Dedicated to Helen Folasade Adu
}

(Received 12 January 2005; revised 27 July 2005)

Communicated by A. J. Pryde

\begin{abstract}
This article concerns the uniform classification of infinite dimensional real topological vector spaces. We examine a recently isolated linearization procedure for uniform homeomorphisms of the form $\phi: X \rightarrow Y$, where $X$ is a Banach space with non-trivial type and $Y$ is any topological vector space. For such a uniform homeomorphism $\phi$, we show that $Y$ must be normable and have the same supremal type as $X$. That $Y$ is normable generalizes theorems of Bessaga and Enflo. This aspect of the theory determines new examples of uniform non-equivalence. That supremal type is a uniform invariant for Banach spaces is essentially due to Ribe. Our linearization approach gives an interesting new proof of Ribe's result.
\end{abstract}

2000 Mathematics subject classification: primary 46B20; secondary 46B04.

\section{Introduction}

Enflo [7, Theorem 6.2.1] proved that if a quasi-normed space $Y$ is uniformly homeomorphic to a Banach space $X$ with 'roundness' $p>1$, then there is a norm on $Y$ that is uniformly equivalent to the given quasi-norm. Prassidis and Weston [12, Theorem 4.2] generalized this theorem to include all Banach spaces $X$ of non-trivial type. In so doing, they isolated from Enflo's earlier work a linearization procedure that applies, in particular, to any uniform homeomorphism between Banach spaces of non-trivial type. The purpose of this article is to examine this linearization procedure in more depth and to extend its range to include all topological vector spaces.

More specifically, we show in Theorems 6.1 and 6.2 that if a topological vector space $Y$ is uniformly homeomorphic to a Banach space $X$ that has non-trivial type, then

(C) 2007 Australian Mathematical Society 1446-7887/07 \$A2.00+0.00 
$Y$ must be normable and have the same supremal type as $X$. That $Y$ is normable generalizes theorems of Bessaga [3] and Enflo [7]. This aspect of the theory leads to new results and insights into the uniform structure of spaces such as $L_{0}(\mu)$. That supremal type is a uniform invariant for Banach spaces is essentially due to Ribe [13]. Our proof of Ribe's result, via remodelling and linearization, is obtained in a completely different manner to existing arguments in the literature. Several other classical results in the uniform theory of topological vector spaces are also shown to be derivable from this one very general linearization procedure.

It should be emphasized that there is a paucity of linearization techniques in the uniform theory of Banach spaces, and not without reason. This is because uniformly continuous functions do not have derivatives in general. The situation is, for example, quite different for Lipschitz homeomorphisms, as can be seen from the survey article by Yost [16]. Note also the text by Sims [15, Chapter 14].

All vector spaces in this article are assumed real and infinite dimensional.

\section{Linear and non-linear type}

The importance and rôle of Rademacher (or, linear) type in the contemporary understanding of the geometry of Banach spaces is exceptionally well documented. See, for example, Pisier [11]. In Bourgain, Milman and Wolfson [4], a notion of non-linear type for general metric spaces was developed, generalizing linear type in Banach spaces. They showed that the supremum of the linear types of a Banach space is equal to the supremum of its metric types. Pisier [11] provides a simplified proof of this theorem. In this article it will be very helpful to work with metric type (rather than linear type), and we recall its definition now.

An $n$-cube $C$ in a metric space $(X, d)$ is a collection of $2^{n}$ points $x_{\bar{t}} \in X$ where each point is indexed by a unique $n$-vector $\vec{t}=(t(1), t(2), \ldots, t(n)) \in\{0,1\}^{n}$. The indexing vectors allow one to identify diagonals and edges in the cube. Specifically, a diagonal in $C$ is an unordered pair of vertices $\left(x_{\bar{u}}, x_{\bar{v}}\right)$ with $u(i) \neq v(i)$ for all $i \in\{1,2, \ldots, n\}$. An edge in $C$ is an unordered pair of vertices $\left(x_{\vec{w}}, x_{\bar{z}}\right)$ with $w(i) \neq z(i)$ for precisely one $i \in\{1,2, \ldots, n\}$. Throughout this article, given an $n$-cube $C$, we will use $D(C)$ and $E(C)$ to denote the sets of all diagonals and all edges in $C$, respectively.

DEFINITION 2.1. Let $p \geq 1$. A metric space $(X, d)$ has metric type $p$ if there is a constant $\mathscr{B}>0$ such that for all $n \in \mathbb{N}$ and all $n$-cubes $C=\left(x_{i}\right)$ in $X$ we have

$$
\left(\sum_{\left(x_{\bar{u}}, x_{\bar{v}}\right) \in D(C)} d\left(x_{\bar{u}}, x_{\bar{v}}\right)^{2}\right)^{1 / 2} \leq \mathscr{B} n^{1 / p-1 / 2}\left(\sum_{\left(x_{\bar{w}}, x_{\bar{z}}\right) \in E(C)} d\left(x_{\bar{w}}, x_{\bar{z}}\right)^{2}\right)^{1 / 2} .
$$


It is noteworthy that the notions of linear and non-linear type were predated by Enflo's [5] notion of roundness. We will not discuss roundness here except to say that it can be directly figured as an analogue of non-linear type.

\section{Uniform Banach groups}

The notion of a uniform Banach group generalizes the additive group structure of a Banach space. It is a group structure on a Banach space that satisfies some compatibility conditions with the norm. Uniform Banach groups were introduced by Enflo in [6] and [7].

Definition 3.1. Let $(X,\|\cdot\|)$ be a Banach space. Suppose that the map $X \times X \rightarrow$ $X:(x, y) \mapsto x \cdot y$ is a group operation on $X$ that is uniformly continuous as a function of two variables with identity $0=0_{X}$, the zero vector of $X$. Then the resulting group structure $G=(X, \cdot)$ is said to be a uniform Banach group modelled on $(X,\|\cdot\|)$. We will often write $x y$ instead of $x \cdot y$.

Uniform homeomorphisms generate examples of uniform Banach groups in the following way: suppose that $X$ is a Banach space, $Y$ is a topological vector space, and $\phi: X \rightarrow Y$ is a uniform homeomorphism such that $\phi(0)=0$. Then, the product $x \cdot y=\phi^{-1}(\phi(x)+\phi(y))$ defines a commutative uniform Banach group structure $G=(X, \cdot)$ that is modelled on $X$. We say that $G$ is the uniform Banach group induced by $\phi$. Through a deep analysis of such structures, Enflo [7] deduced that $L_{p}[0,1]$ is not uniformly homeomorphic to $L_{q}[0,1]$ if $0<q<1<p<\infty$.

There are no known examples of non-commutative uniform Banach groups.

\section{4. $G$-invariant metrics}

Suppose that $G=(X, \cdot)$ is a commutative uniform Banach group modelled on $(X,\|\cdot\|)$. We can introduce a $G$-invariant metric $d$ on $X$ as follows:

$$
d(x, y)=\sup _{w \in X}\|w x-w y\|, \quad x, y \in X .
$$

Obviously $d(x, y) \geq\|x-y\|$ for all $x, y \in X$. This $G$-invariant metric $d$ is uniformly equivalent to the $\|\cdot\|$-distance. In other words, the identity map $(X,\|\cdot\|) \rightarrow(X, d)$ : $x \mapsto x$ is a uniform homeomorphism. As $d$ is a $G$-invariant metric on $X$, we get a triangle inequality of the form $d(x y, 0) \leq d(x, 0)+d(y, 0)$ for all $x, y \in X$. Associated with the $G$-invariant metric $d$ is a chain or intrinsic metric $d_{l}$ defined in the following way. Let $x, y \in X$ be given. Any finite sequence $x=x_{0}, x_{1}, \ldots, x_{n}=y$ 
$\left(n \in \mathbb{N}\right.$ ) of points in $X$ such that $d\left(x_{i}, x_{i+1}\right) \leq 1$ for all $i, 0 \leq i \leq n-1$, is called a one-chain between $x$ and $y$. The intrinsic or chain distance between $x$ and $y$ is given by

$$
d_{I}(x, y)=\inf \sum_{i=0}^{n-1} d\left(x_{i}, x_{i+1}\right),
$$

where the infimum is taken over all one-chains $x=x_{0}, \ldots, x_{n}=y$ between $x$ and $y$.

For all $x, y \in X$ it is clear that $\|x-y\| \leq d(x, y) \leq d_{I}(x, y)$ and, moreover, that $d_{I}(x, y)=d(x, y)$ if (additionally) $d(x, y) \leq 1$. It follows that the chain metric $d_{I}$ is also uniformly equivalent to the $\|\cdot\|$-distance.

\section{5. $G$-invariant metrics and the (partial) stability of type}

The following lemma is standard to the uniform theory of Banach spaces and is given without proof.

LeMMA 5.1. Let $(X,\|\cdot\|)$ be a Banach space. Let $\rho$ be any metric on $X$ that is uniformly equivalent to the $\|\cdot\|$-distance. Then there is a positive constant $\mathscr{C}$ such that $\rho(x, y) \leq \mathscr{C}\|x-y\|$ whenever $\|x-y\| \geq 1$ or $\rho(x, y) \geq 1$.

The next theorem shows that if $(X,\|\cdot\|)$ and $(X, \rho)$ satisfy the hypotheses of Lemma 5.1 and if $\rho$ dominates the $\|\cdot\|$-distance, then $(X, \rho)$ will inherit the metric types of $(X,\|\cdot\|)$ at 'large distances'. There does not seem to be any obvious way to extend this statement to include 'small distances' and, indeed, it might not hold under these circumstances.

THEOREM 5.2. Let $(X,\|\cdot\|)$ be a Banach space. Suppose $(X,\|\cdot\|)$ has metric type $p$ with type constant $\mathscr{B}$. Let $\rho$ be any metric on $X$, uniformly equivalent to the $\|\cdot\|$-distance, that satisfies $\|x-y\| \leq \rho(x, y)$ for all $x, y \in X$. Then there is a constant $\mathscr{C}>0$ such that

$$
\left(\sum_{D(C)} \rho\left(x_{\vec{u}}, x_{\vec{v}}\right)^{2}\right)^{1 / 2} \leq \mathscr{C} \mathscr{B} n^{1 / p-1 / 2}\left(\sum_{E(C)} \rho\left(x_{\vec{w}}, x_{\bar{z}}\right)^{2}\right)^{1 / 2}
$$

for any $n$-cube $C=\left(x_{i}^{-}\right)$in $X$ whose diagonals all have $\|\cdot\|$-length (and/or, $\rho$-length) at least one.

ProOF. Lemma 5.1 gives a constant $\mathscr{C}>0$ such that $\rho(x, y) \leq \mathscr{C} \cdot\|x-y\|$ whenever $\|x-y\| \geq 1$ or $\rho(x, y) \geq 1$. Fix an $n$-cube $C=\left(x_{i}\right)$ in $X$ whose diagonals all have $\|\cdot\|$-length (and/or $\rho$-length) at least one. Using Lemma 5.1, together with 
the hypotheses on $(X,\|\cdot\|)$ and $\rho$, we see that

$$
\begin{aligned}
\left(\sum_{D(C)} \rho\left(x_{\vec{u}}, x_{\vec{v}}\right)^{2}\right)^{1 / 2} & \leq \mathscr{C}\left(\sum_{D(C)}\left\|x_{\vec{u}}-x_{\vec{v}}\right\|^{2}\right)^{1 / 2} \\
& \leq \mathscr{C} \mathscr{B} n^{1 / p-1 / 2}\left(\sum_{E(C)}\left\|x_{\vec{w}}-x_{\bar{z}}\right\|^{2}\right)^{1 / 2} \\
& \leq \mathscr{C} \mathscr{B} n^{1 / p-1 / 2}\left(\sum_{E(C)} \rho\left(x_{\vec{w}}, x_{\bar{z}}\right)^{2}\right)^{1 / 2}
\end{aligned}
$$

In the case of a commutative uniform Banach group $G=(X, \cdot), \rho$ could be either of the $G$-invariant metrics $d$ or $d_{l}$ introduced in Section 4. This is how we will use Theorem 5.2 in the next section.

\section{Linearization of certain uniform homeomorphisms}

Every Banach space has (metric) type 1. In the statement of Theorem 6.1 (below) the essential hypothesis is on the Banach space $X$. Namely, that it has non-trivial (metric) type $p>1$. The assumption that $Y$ is a Banach space can be weakened considerably. This is done in Theorem 6.2. The technique being developed in this article is seen to have two important aspects: $Y$ is not restricted to be a normed (or, even quasi-normed) space and (appropriately) the Corson-Klee Lemma is not used.

THEOREM 6.1. Let $p>1$. Let $(X,\|\cdot\|)$ be a Banach space with metric type $p$. If $(X,\|\cdot\|)$ is uniformly homeomorphic to a Banach space $(Y,\|\cdot\|)$, then the vector space operations and norm on $X$ can be re-modelled (in a uniformly equivalent way) to produce a new Banach space $(X, N(\cdot))$ that has metric type $p$, and which is, moreover, linearly isomorphic to $(Y,\|\cdot\|)$. In particular, $Y$ is seen to have metric type $p$.

ProOF. Denote by $\mathscr{B}$ the metric type $p$ constant of $(X,\|\cdot\|)$. We may assume that the uniform homeomorphism $\phi:(X,\|\cdot\|) \rightarrow(Y,\|\cdot\|)$ satisfies $\phi(0)=0$. Denote by $G=(X, \cdot)$ the uniform Banach group modelled on $(X,\|\cdot\|)$ that is induced by $\phi$. So, for all $x, y \in X, x y=\phi^{-1}(\phi(x)+\phi(y))$. Introduce the $G$-invariant metrics $d$ and $d_{I}$ associated with this group structure. For any $x \in X$ and $t \in \mathbb{R}$, define $x^{t}=\phi^{-1}(t \phi(x)$. Introduce new vector space operations on $X$ as follows: addition is the group multiplication $(x, y) \mapsto x y$, and scalar multiplication of $x \in X$ by $t \in \mathbb{R}$ is given by $x^{t}$. For all $x \in X$, define

$$
N(x)=\limsup _{t \rightarrow \infty} \frac{d_{I}\left(x^{t}, 0\right)}{t} .
$$


Prassidis and Weston [12, Theorem 4.2] showed that $N(\cdot)$ is a norm relative to the new vector space operations on $X$, and that

$$
\|x\| \leq N(x) \leq d_{I}(x, 0)
$$

for all $x \in X$. For brevity we write $(X, N(\cdot))$ to denote this new normed vector space structure. A simple (omitted) argument shows that $(X, N(\cdot))$ is complete. Since $d_{l}$ is uniformly equivalent to the original $\|\cdot\|$-distance, it follows from (1) that the identity map $(X,\|\cdot\|) \rightarrow(X, N(\cdot)): x \mapsto x$ is a (not necessarily linear) uniform homeomorphism. Hence, by virtue of the new vector space operations in place on $X$, we see that $\phi:(X, N(\cdot)) \rightarrow(Y,\|\cdot\|)$ is a linear uniform homeomorphism. Put differently, as a map from $(X, N(\cdot))$ to $(Y,\|\cdot\|), \phi$ is a linear isomorphism.

It remains to show that $(X, N(\cdot))$ has metric type $p$. Applied to the original Banach space $(X,\|\cdot\|)$ and the $G$-invariant metric $d_{I}$ on $X$, Lemma 5.1 gives a constant $\mathscr{C}>0$ such that $d_{I}(x, y) \leq \mathscr{C}\|x-y\|$ whenever $\|x-y\| \geq 1$ or $d_{I}(x, y) \geq 1$. Importantly, relative to this constant $\mathscr{C}$, the conclusion of Theorem 5.2 holds for the pair of spaces $(X,\|\cdot\|)$ and $\left(X, d_{I}\right)$. This will be used below.

The metric $d_{I}$ is also uniformly equivalent to the new norm $N(\cdot)$ and satisfies $N\left(x y^{-1}\right) \leq d_{l}\left(x y^{-1}, 0\right)=d_{l}(x, y)$ for all $x, y \in X$ by $G$-invariance. Hence by Lemma 5.1, applied to the pair of spaces $(X, N(\cdot))$ and $\left(X, d_{l}\right)$, there is a constant $\mathscr{D}>0$ such that

$$
d_{I}(x, y)=d_{I}\left(x y^{-1}, 0\right) \leq \mathscr{D} N\left(x y^{-1}\right)
$$

for all $x, y \in X$ with $N\left(x y^{-1}\right) \geq 1$.

To determine that $(X, N(\cdot))$ has metric type $p$ (with type constant $\mathscr{C} \mathscr{B} \mathscr{D}$ ), it suffices to consider only those $n$-cubes $C=\left(x_{i}\right)$ in $X$ whose non-zero $N(\cdot)$-distances are all at least one. This is because $N(\cdot)$ is scalar homogeneous. Take such an $n$-cube $C=\left(x_{i}\right)$ in $X$. Then by using (1), Theorem 5.2 (as above), and (2) (consecutively), we see that

$$
\begin{aligned}
\left(\sum_{D(C)} N\left(x_{\bar{u}} x_{\vec{v}}^{-1}\right)^{2}\right)^{1 / 2} & \leq\left(\sum_{D(C)} d_{I}\left(x_{\vec{u}}, x_{\vec{v}}\right)^{2}\right)^{1 / 2} \\
& \leq \mathscr{C} \mathscr{B} n^{1 / p-1 / 2}\left(\sum_{E(C)} d_{I}\left(x_{\bar{w}}, x_{\bar{z}}\right)^{2}\right)^{1 / 2} \\
& \leq \mathscr{C} \mathscr{B} \mathscr{D} n^{1 / p-1 / 2}\left(\sum_{E(C)} N\left(x_{\vec{w}} x_{\bar{z}}^{-1}\right)^{2}\right)^{1 / 2} .
\end{aligned}
$$

We conclude that $(X, N(\cdot))$, and therefore $(Y,\|\cdot\|)$, has metric type $p$. 
As noted in the preamble at the beginning of this section, the hypothesis on $Y$ in the statement of Theorem 6.1 can be greatly relaxed. In fact, the next theorem determines that $Y$ need only be a topological vector space.

THEOREM 6.2. Let $Y$ be a topological vector space. If $Y$ is uniformly homeomorphic to a closed subspace of a Banach space $(X,\|\cdot\|)$ that has non-trivial type, then the topology of $Y$ is given by a norm.

Proof. The properties of non-trivial type $p>1$ and completeness are inherited by the closed subspaces of $(X,\|\cdot\|)$. So, without loss of generality, it suffices to consider a uniform homeomorphism $\phi:(X,\|\cdot\|) \rightarrow Y$ with $\phi(0)=0$. The same argument as given in the proof of Theorem 6.1 constructs the remodelled Banach space $(X, N(\cdot))$ that is induced by the map $\phi$. And again, as a map from the Banach space $(X, N(\cdot))$ to the topological vector space $Y, \phi$ is a linear uniform homeomorphism. In particular, as $X$ is metrizable, this implies that the map $\phi:(X, N(\cdot)) \rightarrow Y$ is bounded. See, for example, Rudin [14, Theorem 1.32]. It follows from the Open Mapping Theorem (see, for example, Rudin [14, Theorem 2.11]) that $Y$ is an $F$-space. Therefore, the map $\phi^{-1}: Y \rightarrow(X, N(\cdot))$ is also bounded via Rudin [14, Theorem 1.32]. However, an $F$-space that is linearly isomorphic to a Banach space is obviously locally convex and locally bounded. Therefore $Y$ is normable.

It should be noted that Theorem 6.2 is due to Bessaga [3] in the case that $Y$ is assumed to be a locally convex topological vector space. In fact, Bessaga [3] showed that if a locally convex topological vector space is uniformly homeomorphic to a normed space, then it is normable. Many new examples of uniform non-equivalence can be deduced (automatically) from Theorem 6.2. One such result, that stands out in relation to the uniform classification of $L_{p}$-spaces, is the following.

COROLlaRY 6.3. If $X$ is a Banach space that has non-trivial type, then $L_{0}(\mu)$ is not uniformly homeomorphic to any (closed) subspace of $X$.

In contrast to Corollary 6.3, Aharoni, Maurey and Mitjagin [2] have shown that $L_{0}(\mu)$ is, however, uniformly homeomorphic to a subset of Hilbert space. See also Aharoni [1] for related results on uniform equivalence to subsets of $L_{p}, 1 \leq p$.

Given Banach spaces $X$ and $Y$ of non-trivial (metric) type, and a uniform homeomorphism $\phi: X \rightarrow Y$ (that may be assumed to satisfy $\phi(0)=0$ ), the linearization procedure described in Theorem 6.1 (and its proof) is clearly reversible. We can apply the same method to $Y$ and $\phi^{-1}$ to produce a new Banach space $(Y, M(\cdot))$ that is linearly isomorphic to $X$ and has the same metric types as $Y$. Hence the supremum of the types of $X$ (which is equal to the supremum of its metric types, by Bourgain, Milman and Wolfson [4]) equals the supremum of the types of $Y$. Moreover, as type is inherited 
by subspaces, this same argument extends to any (closed) subspace $Z$ of $Y$ that may also happen to be uniformly homeomorphic to $X$. In summary, we have determined a new proof of the following classical result in the uniform theory of Banach spaces, a result that is essentially due to Ribe [13].

COROLlaRY 6.4. Let $p>1$. Let $X, Y$ be Banach spaces. Suppose $X$ is uniformly homeomorphic to a (closed) subspace $Z$ of $Y$. Then $X$ has type $p$ if and only if $Z$ has type p. Put differently, supremal type is a uniform invariant for Banach spaces.

Other classical results in the uniform theory of Banach spaces can be deduced from Theorems 6.1-6.2 and Corollary 6.4. We give one such example. In the commutative setting, this result is due to Lindenstrauss [10] when $q \geq 1$ and $\max (p, q)>2$, and to Enflo [5, 6] when $\max (p, q) \leq 2$. The result is new for $q=0$ (and follows from Corollary 6.3). The corollary as stated is seen to follow from Theorems 6.1-6.2 and Corollary 6.4 via standard facts about the type and local convexity of $L_{p}$-spaces.

COROLLARY 6.5. Let $p, q$ be distinct non-negative real numbers with $0 \leq q<p$. If $1 \leq \min (p, q) \leq 2$, or if $0 \leq q<1<p$, then $L_{q}(\mu)$ is not uniformly homeomorphic to any (closed) subspace of $L_{p}(v)$.

Type computations of Fack [8] imply that Corollary 6.5 remains valid if $L_{p}$ and $L_{q}$ are non-commutative $L_{r}$-spaces, possibly corresponding to different von Neumann algebras. For $1 \leq \min (p, q)$, this non-commutative version of Corollary 6.5 was previously noted by Lennard, Tonge and Weston [9]. For $q<1$ the non-commutative result is explicitly noted for the first time. One may also formulate variants of Corollary 6.5 for large classes of (subspaces of) Orlicz and Lorentz spaces based on considerations of their type or local convexity. However, we will not do so here.

\section{Acknowledgments}

This research was supported by a generous Canisius College 'Summer Faculty Research Fellowship'. Special thanks to Christopher Lennard, Petrus Potgieter, Efstratios Prassidis and Andrea Spain for the helpful comments they made during the preparation of this article.

\section{References}

[1] I. Aharoni, 'Uniform embeddings of Banach spaces', Israel J. Math. 27 (1977), 174-179.

[2] I. Aharoni, B. Maurey and B. S. Mitjagin, 'Uniform embeddings of metric spaces and of Banach spaces into Hilbert spaces', Israel J. Math. 52 (1985), 251-265. 
[3] C. Bessaga, 'On topological classification of complete linear metric spaces', Fund. Math. 56 (1965), 251-288.

[4] J. Bourgain, V. Milman and H. Wolfson, 'On type of metric spaces', Trans. Amer. Math. Soc. 294 (1986), 295-317.

[5] P. Enflo, 'On the nonexistence of uniform homeomorphisms between $L_{p}$-spaces', Ark. Mat. 8 (1968), 103-105.

[6] — ' 'Uniform structures and square roots in topological groups I', Israel J. Math. 8 (1970), $230-252$.

[7] —_, 'Uniform structures and square roots in topological groups II', Israel J. Math. 8 (1970), 253-272.

[8] T. Fack, 'Type and cotype inequalities for non commutative $L^{p}$ spaces', J. Operator Theory 17 (1987), 255-279.

[9] C. J. Lennard, A. M. Tonge and A. Weston, 'Roundness and metric type', J. Math. Anal. Appl. 252 (2000), 980-988.

[10] J. Lindenstrauss, 'On nonlinear projections in Banach spaces', Michigan Math. J. 11 (1964), 263-287.

[11] G. Pisier, Probabilistic methods in the geometry of Banach spaces Lecture Notes in Math. 1206 (Springer-Verlag, New York, 1986) pp. 167-241.

[12] E. Prassidis and A. Weston, 'Uniform Banach groups and structures', C. R. Math. Acad. Sci. Canada 26 (2004), 25-32.

[13] M. Ribe, 'On uniformly homeomorphic normed spaces', Ark. Mat. 14 (1976), 237-244.

[14] W. Rudin, Functional analysis, McGraw-Hill International Series in Pure and Applied Mathematics, 2nd edition (McGraw-Hill, New York, 1991).

[15] B. Sims, "Ultra"-techniques in Banach space theory, Queen's Papers in Pure and Applied Mathematics 60 (Queens University, Kingston, 1982).

[16] D. Yost, 'If every doughnut is a teacup, then every Banach space is a Hilbert space', Seminar on Functional Analysis, Universidad de Murica, Notas de Matematica 1 (1987), 125-148.

Department of Mathematics \& Statistics

Canisius College

Buffalo, NY 14208

USA

e-mail: westona@canisius.edu 